\title{
Distorted-wave calculation of cross sections for inner-shell ionization by electron and positron impact
}

\author{
Silvina Segui, Michael Dingfelder, and Francesc Salvat* \\ Facultat de Física (ECM), Universitat de Barcelona, Diagonal 647, 08028 Barcelona, Spain
}

(Received 7 March 2003; published 24 June 2003)

\begin{abstract}
The relativistic distorted-wave Born approximation is used to calculate differential and total cross sections for inner shell ionization of neutral atoms by electron and positron impact. The target atom is described within the independent-electron approximation using the self-consistent Dirac-Fock-Slater potential. The distorting potential for the projectile is also set equal to the Dirac-Fock-Slater potential. For electrons, this guarantees orthogonality of all the orbitals involved and simplifies the calculation of exchange $T$-matrix elements. The interaction between the projectile and the target electrons is assumed to reduce to the instantaneous Coulomb interaction. The adopted numerical algorithm allows the calculation of differential and total cross sections for projectiles with kinetic energies ranging from the ionization threshold up to about ten times this value. Algorithm accuracy and stability are demonstrated by comparing differential cross sections calculated by our code with the distorting potential set to zero with equivalent results generated by a more robust code that uses the conventional plane-wave Born approximation. Sample calculation results are presented for ionization of $K$ - and $L$-shells of various elements and compared with the available experimental data.
\end{abstract}

DOI: 10.1103/PhysRevA.67.062710

PACS number(s): 34.80.Dp

\section{INTRODUCTION}

Knowledge of accurate cross sections for ionization of inner atomic electron shells by electron impact is required for a quantitative analysis with various spectroscopic techniques such as Auger-electron spectroscopy, electron probe microanalysis (EPMA), and electron energy-loss spectroscopy. These cross sections are also needed for the description of the energy spectra of $\mathrm{x}$-rays from sources used in $\mathrm{x}$ ray fluorescence analysis and for medical or industrial diagnosis. Currently, there is a practical demand for accurate electron/ (positron) interaction data for Monte Carlo simulation of radiation transport in matter, which is of application in multiple fields, including the aforesaid analytical and control techniques as well as detector response studies, radiation therapy, and dosimetry.

Unfortunately, a systematic method for calculating electron impact ionization cross sections for atoms from first principles has not yet been generally agreed upon. Calculations within the plane-wave first-order Born approximation (PWBA) provide reliable energy-loss differential ionization cross sections and integrated (total) cross sections for highenergy electrons. The accuracy of the PWBA deteriorates progressively when the kinetic energy of the projectile decreases towards the ionization threshold, because of the increasing distorting effect of the atomic field on the incident and emerging waves and, in the case of projectile electrons, because of exchange effects. As an alternative to more accurate first-principles calculations, semiempirical modifications of the PWBA have been proposed to account for these effects $[1,2]$. Furthermore, empirical and semiempirical analytical cross-section formulas have been proposed for practical use [3-5]. Most of these formulas only yield the total ionization

\footnotetext{
*Author to whom correspondence should be addressed.
}

cross section and are based on existing experimental data, which are limited both in number and in accuracy (see below).

Recent experimental and theoretical studies of the triply differential cross section for $K$-shell ionization by impact of relativistic electrons [6] have shown that the PWBA is clearly insufficient to describe these interactions. To get a quantitative agreement with the experiments, calculations must be performed within the distorted-wave first-order Born approximation (DWBA), in which the initial and final projectile wave functions include the distortion caused by the atomic field, also allowing the description of exchange effects in a consistent way. DWBA calculations for the excitation of multiply charged ions have been described by various authors $[7,8]$, and applied to generate systematic numerical tables and analytical approximations for the total ionization cross section of ions. To the best of our knowledge, similar systematic calculations for the ionization of neutral atoms do not exist. It is also worth mentioning that the studies of Keller and co-workers [6] demonstrate the reliability of the DWBA for "hard" interactions of fast projectiles, involving large energy transfers for which the two free electrons after the collision have relatively high energies. It is then of fundamental interest to investigate the reliability of the DWBA in the complementary regime of "soft" collisions and projectiles with low, near threshold energies.

The lack of systematic DWBA calculations for inner-shell ionization of neutral atoms is mostly due to the extremely slow convergence of the partial-wave expansions for energetic particles. Note that the calculations involve distortedwave functions of fast free electrons (the initial energy of the projectile is larger than the ionization energy of the active shell), which oscillate rapidly over ample space volumes. A related difficulty is that single-particle wave functions must be calculated to high accuracy to ensure that accumulated errors from the multiple integrals and sums will not completely hide the final numerical value. Nevertheless, with 
currently available computational power and numerical techniques, DWBA calculations of neutral atom inner-shell ionization by charged particle impact are feasible, at least for limited energy and energy-loss ranges.

In the present paper, we describe a relatively simple physical model and a robust calculation algorithm that we have developed to compute the DWBA energy-loss differential cross sections (DCSs) for ionization of inner shells of neutral atoms and ions by the impact of electrons and positrons. We are mostly interested in the energy range from the ionization threshold up to, say, $100 \mathrm{keV}$ for which the effective interaction between the projectile and the active target electron shell reduces to the instantaneous Coulomb interaction. An objective of our research is to generate a systematic database of inner-shell ionization cross sections of atoms to be used in practical Monte Carlo simulations of radiation transport and, specifically, for EPMA and x-ray generation studies. In practice, the calculation of DWBA ionization cross sections described here is feasible only for projectile kinetic energies in a limited range, namely, from the ionization threshold up to about ten times this value. For higher kinetic energies, we must content ourselves with a more pragmatic procedure that consists of introducing empirical (Coulomb and exchange) corrections to the PWBA. Ideally, these corrections should have a negligible effect on the DCSs at high energies and reproduce the DWBA results near the ionization threshold. Work along these lines is in progress and will be presented elsewhere [9].

A comment on the experimental information available to validate theoretical calculations is in order. Measured total cross sections for $K$-shell ionization published prior to 1990 were compiled by Long et al. [10]. Since then, additional measurements for $K$ shells have been reported [11-16]. An inspection of the currently available experimental data reveals that these are still scarce for many elements and, when these are available, one usually finds significant discrepancies between data from different authors. These discrepancies are often much larger than the stated experimental uncertainties, reflecting the fact that measurements of ionization cross sections face considerable experimental difficulties. The situation for $L$ and outer shells is even less satisfactory. In the analysis of our theoretical results, we shall heavily rely on total cross sections measured by Llovet et al. [15] and Campos et al. [16], mostly because these are affected by relative uncertainties much smaller than data from other sources. These authors give the "shape" of the cross section versus energy curve with a relative accuracy of $\sim 3 \%$; most of the uncertainty in the total cross sections comes from the global energy-independent factor that transforms their relative data ( $\mathrm{x}$-ray intensities) into absolute cross-section values.

The paper is organized as follows. The theoretical framework is sketched in Sec. II. In Sec. III, we describe the potentials adopted in the calculations. The numerical methods employed to solve the radial Dirac equations and to sum the partial-wave series are described in Sec. IV, which also contains an analysis of the accuracy and stability of the whole calculation. In Sec. V, total ionization cross sections obtained from the proposed DWBA method are compared with the

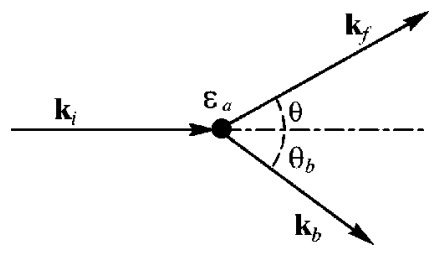

FIG. 1. Kinematics of the interaction.

available experimental data for electron and positron ionizing collisions with various elements and different shells, and some concluding remarks are given.

\section{THEORY}

We shall briefly formulate a semirelativistic version of the DWBA for inelastic collisions of electrons and positrons, i.e., particles with spin $\frac{1}{2}$, mass $m_{\mathrm{e}}$, and charge $Z_{0} e\left(Z_{0}=\right.$ $\mp 1$ ) with an atom or ion of the element $Z$ (= atomic number) having $N$ electrons in its ground state. Although we are mainly concerned with collisions of electrons and positrons, the formal theory is applicable to particles with other masses and charges. We will assume that the mass of the target atom is infinite, which is a good approximation when the projectile is an electron or positron, and compute the cross sections in the laboratory reference frame, where the target is at rest. Figure 1 displays the kinematics of the collision. Before the interaction, the projectile moves with velocity $\mathbf{v}_{i}$, linear momentum $\mathbf{p}_{i}=\hbar \mathbf{k}_{i}$, and kinetic energy $\epsilon_{i}$. The corresponding values after the collision are $\mathbf{v}_{f}, \mathbf{p}_{f}=\hbar \mathbf{k}_{f}$ and $\epsilon_{f}$, respectively. The ionized electron is ejected with energy $\epsilon_{b}$ and momentum $\mathbf{p}_{b}=\hbar \mathbf{k}_{b}$.

In the Coulomb gauge, the effective interaction $\mathcal{H}_{\text {int }}(0,1)$ between a charged Dirac particle "0" and an electron "1" can be expressed as [17]

$$
\begin{aligned}
\mathcal{H}_{\text {int }}(0,1)= & -\frac{Z_{0} e^{2}}{\left|\mathbf{r}_{1}-\mathbf{r}_{0}\right|}+\frac{Z_{0} e^{2}}{2 \pi^{2}} \int d \mathbf{q} \frac{\boldsymbol{\alpha}_{0} \cdot \boldsymbol{\alpha}_{1}-\left(\boldsymbol{\alpha}_{0} \cdot \hat{\mathbf{q}}\right)\left(\boldsymbol{\alpha}_{1} \cdot \hat{\mathbf{q}}\right)}{q^{2}-(W / \hbar c)^{2}} \\
& \times \exp \left[\mathrm{iq} \cdot\left(\mathbf{r}_{1}-\mathbf{r}_{0}\right)\right],
\end{aligned}
$$

where $\boldsymbol{\alpha}_{0}$ and $\boldsymbol{\alpha}_{1}$ denote Dirac matrices of the projectile and the electron, respectively, and $W$ is the energy exchanged in the interaction. The first term is the instantaneous Coulomb interaction. The second one accounts for the exchange of virtual photons in the lowest non-vanishing perturbation order. This term is usually referred to as the transverse interaction. As the contribution of each $\boldsymbol{\alpha}$ is of the order of $v / c$, where $v$ is the velocity of the particle, the effect of the transverse interaction is appreciable only when the two interacting particles have relativistic speeds. Here, we disregard this part of the interaction and assume that the collisions are appropriately described by the (longitudinal) Coulomb term alone. This sets an upper limit to the energy interval where our DWBA is applicable. For projectiles with higher energies, the complete effective interaction (1) can be considered within the PWBA, which provides a reliable description of inelastic collisions in the high-energy limit. 
In our formulation of the DWBA, the Hamiltonian of the system (projectile + target) is expressed as [18]

$$
\mathcal{H}(0,1, \ldots, N)=\mathcal{H}_{\mathrm{T}}(1, \ldots, N)+\mathcal{H}_{\mathrm{P}}(0)+\mathcal{H}^{\prime}(0,1, \ldots, N),
$$

where $\mathcal{H}_{\mathrm{T}}(1, \ldots, N)$ and $\mathcal{H}_{\mathrm{P}}(0)$ are the "unperturbed" Dirac Hamiltonians of the target and the projectile, respectively. $\mathcal{H}^{\prime}$ is a perturbation Hamiltonian which includes the interaction of the projectile with the nucleus and the atomic electrons,

$$
\mathcal{H}^{\prime}(0,1, \ldots, N)=Z_{0} e \varphi_{\text {nuc }}\left(r_{0}\right)+\sum_{I=1}^{N} \mathcal{H}_{\text {int }}(0, I)-V_{\mathrm{P}}\left(r_{0}\right) .
$$

Here, $V_{\mathrm{P}}\left(r_{0}\right)$ is the distorting central potential "seen" by the projectile, which should be chosen in such a way that $\mathcal{H}^{\prime}$ can be treated as a first-order perturbation. Evidently, within this model, the eigenstates of the unperturbed Hamiltonian of the total system can be factorized in the form $\psi(0) \Psi_{n}(1, \ldots, N)$. To facilitate numerical computations, the states of the target atom are described by using an independent-electron approximation, i.e., atomic electrons are considered to move independently in a common central field $V_{\mathrm{T}}(r)$, which is assumed to describe both the initial and the final atomic states. Hence, these states can be represented as single Slater determinants, $\Psi_{n}=\operatorname{det}\left[\psi_{a}\right]$, made up of $N$ orbitals $\psi_{a}$ that are solutions of the Dirac equation for the potential $V_{\mathrm{T}}(r)$ and are, therefore, mutually orthogonal. This also ensures the orthogonality of the atomic states $\Psi_{n}$. The projectile wave functions $\psi_{i}\left(\mathbf{r}_{0}\right)$ satisfy the Dirac equation for the distorting field $V_{\mathrm{P}}\left(r_{0}\right)$.

In this approximation, the only allowed transitions of the target atom are single-electron excitations, i.e., the interaction causes excitations of the target atom from the initial state $\Psi_{a}$ (usually the ground state) to a final state $\Psi_{b}$, which differs from $\Psi_{a}$ by a single orbital. This is equivalent to the so-called one-active-electron approximation, which consists of considering only the excitations of a single electron from a bound orbital $\psi_{a}$ to an unoccupied (bound or free) orbital $\psi_{b}$, whereas the other atomic electrons behave as mere spectators and their orbitals remain frozen in the course of the interaction. Thus, the $T$-matrix element that describes the transitions from an initial state $\psi_{i} \Psi_{a}$ to a final state $\psi_{f} \Psi_{b}$ is expressed as

$$
\begin{aligned}
T_{f i} & =\left\langle\psi_{f}(0) \Psi_{b}(1, \ldots, N)\left|\mathcal{H}^{\prime}(0,1, \ldots, N)\right| \psi_{i}(0) \Psi_{a}(1, \ldots, N)\right\rangle \\
& =\left\langle\psi_{f}(0) \psi_{b}(1)\left|\mathcal{H}_{\text {int }}(0,1)\right| \psi_{i}(0) \psi_{a}(1)\right\rangle,
\end{aligned}
$$

where " 1 " identifies the active target electron. Formally, the theory can be liberated from the one-active-electron approximation by summing the interactions of the projectile with the remaining $N-1$ atomic electrons and using generic atomic wave functions. In the case of ionization of inner closed shells, the use of more elaborate atomic wave functions would have a negligible impact on the calculated cross sections. In practice, the one-active-electron approximation yields a reasonably accurate description of the excitation/ (ionization) of inner (tightly bound) shells of atoms and ions.

We use bound orbitals of the form [19]

$$
\psi_{n \kappa m}(\mathbf{r})=\frac{1}{r}\left(\begin{array}{c}
P_{n \kappa}(r) \Omega_{\kappa m}(\hat{\mathbf{r}}) \\
\mathrm{i} Q_{n \kappa}(r) \Omega_{-\kappa m}(\hat{\mathbf{r}})
\end{array}\right),
$$

where $\Omega_{\kappa m}$ are spherical spinors. Here $n$ is the principal quantum number and $\kappa$ is the relativistic angular momentum quantum number, which is related to the orbital and total angular momentum quantum numbers, $\ell$ and $j=\ell \pm \frac{1}{2}$, through

$$
\kappa=(\ell-j)(2 j+1)
$$

The orbitals (5) are solutions of the one-electron Dirac equation

$$
\left[c \boldsymbol{\alpha} \cdot \mathbf{p}+(\beta-1) m_{\mathrm{e}} c^{2}+V_{\mathrm{T}}(r)\right] \psi_{n \kappa m}(\mathbf{r})=\boldsymbol{\epsilon}_{n \kappa} \psi_{n \kappa m}(\mathbf{r}) .
$$

The radial functions $P_{n \kappa}(r)$ and $Q_{n \kappa}(r)$ satisfy the following coupled differential equations:

$$
\begin{gathered}
\frac{d P_{n \kappa}}{d r}=-\frac{\kappa}{r} P_{n \kappa}+\frac{\epsilon_{n \kappa}-V+2 m_{\mathrm{e}} c^{2}}{c \hbar} Q_{n \kappa}, \\
\frac{d Q_{n \kappa}}{d r}=-\frac{\epsilon_{n \kappa}-V}{c \hbar} P_{n \kappa}+\frac{\kappa}{r} Q_{n \kappa},
\end{gathered}
$$

with the boundary conditions $P_{n \kappa}(0)=0$ and $Q_{n \kappa}(0)=0$.

Free states for the projectile, as well as for the ejected electron, are described by distorted plane waves [20] for the corresponding central potential $\left[V_{\mathrm{P}}(r)\right.$ or $\left.V_{\mathrm{T}}(r)\right]$,

$$
\begin{aligned}
\psi_{\mathbf{k} \mu}^{( \pm)}(\mathbf{r})= & \frac{1}{k} \sqrt{\frac{\epsilon+2 m_{\mathrm{e}} c^{2}}{\pi\left(\epsilon+m_{\mathrm{e}} c^{2}\right)}} \sum_{\kappa, m} \mathrm{i}^{\ell} \exp \left( \pm \mathrm{i} \delta_{\epsilon \kappa}\right) \\
& \times\left\{\left[\Omega_{\kappa m}(\hat{\mathbf{k}})\right]^{\dagger} \chi_{\mu}\right\} \psi_{\epsilon \kappa m}(\mathbf{r}),
\end{aligned}
$$

where $\epsilon$ and $\mathbf{k}$ are the kinetic energy and the wave number of the particle, respectively, $\chi_{\mu}$ are Pauli spinors (i.e., eigenvectors of the Pauli spin matrix $S_{z}$ ), 


$$
\chi+1 / 2=\left(\begin{array}{l}
1 \\
0
\end{array}\right), \quad \chi-1 / 2=\left(\begin{array}{l}
0 \\
1
\end{array}\right)
$$

and $\psi_{\epsilon \kappa m}(\mathbf{r})$ are spherical waves of the type

$$
\psi_{\epsilon \kappa m}(\mathbf{r})=\frac{1}{r}\left(\begin{array}{c}
P_{\epsilon \kappa}(r) \Omega_{\kappa m}(\hat{\mathbf{r}}) \\
\mathrm{i} Q_{\epsilon \kappa}(r) \Omega_{-\kappa m}(\hat{\mathbf{r}})
\end{array}\right)
$$

The functions $P_{\epsilon \kappa}(r)$ and $Q_{\epsilon \kappa}(r)$ satisfy Eqs. (7) with the appropriate potential. $\psi_{\mathbf{k} \mu}^{( \pm)}$behaves asymptotically as a plane wave plus a spherical incoming $(-)$ or outgoing $(+)$ distortion. The phase shifts $\delta_{\epsilon \kappa}$ are determined by the large- $r$ behavior of the radial function $P_{\epsilon \kappa}(r)$. With the radial functions normalized in such a way that $P_{\epsilon \kappa}(r)$ oscillates asymptotically with unit amplitude, the distorted waves are normalized on the wave-number scale, i.e.,

$$
\int\left[\psi_{\mathbf{k}^{\prime} \mu^{\prime}}^{( \pm)}\right]^{\dagger} \psi_{\mathbf{k} \mu}^{( \pm)} d \mathbf{r}=\delta\left(\mathbf{k}^{\prime}-\mathbf{k}\right) \delta_{\mu^{\prime} \mu}
$$

where $\delta_{\mu^{\prime} \mu}$ is the Kronecker delta $\left(=1\right.$ if $\mu=\mu^{\prime}$ and $=0$ otherwise).

The DCS, differential in the energy loss $W$, for the excitation of the active electron from the orbital $\psi_{a}$ to a free orbital $\psi_{b}$ with positive energy $\epsilon_{b}$ is given by [21]

$$
\frac{d \sigma_{\text {ion }}}{d W}=\frac{(2 \pi)^{4}}{\hbar v_{i}} k_{b} k_{f} \frac{\epsilon_{f}+m_{\mathrm{e}} c^{2}}{c^{2} \hbar^{2}} \frac{\epsilon_{b}+m_{\mathrm{e}} c^{2}}{c^{2} \hbar^{2}} \iint\left|T_{f i}\right|^{2} d \hat{\mathbf{k}}_{b} d \hat{\mathbf{k}}_{f} .
$$

In most practical cases, the target atoms are randomly oriented, the incident beam is unpolarized and final magnetic and spin states are not distinguished. Under these circumstances, the DCS for ionization of a subshell $\left(n_{a}, \kappa_{a}\right)$ occupied by $q_{a}$ equivalent electrons is obtained by averaging over the initial degenerate magnetic and spin states and summing over final degenerate states. In addition, owing to the orthogonality of the angular functions involved, the integral over $\hat{\mathbf{k}}_{b}$ can be readily evaluated. After this process, the DCS can be expressed as

$$
\begin{aligned}
\frac{d \sigma_{\mathrm{ion}}}{d W}= & q_{a} \frac{(2 \pi)^{4}}{\hbar v_{i}} k_{b} k_{f} \frac{\epsilon_{f}+m_{\mathrm{e}} c^{2}}{c^{2} \hbar^{2}} \frac{\epsilon_{b}+m_{\mathrm{e}} c^{2}}{c^{2} \hbar^{2}} \frac{1}{2\left[j_{a}\right]} \\
& \times \sum_{m_{a}, m_{\mathrm{S} i}} \sum_{m_{\mathrm{S} f}} \sum_{\kappa_{b}, m_{b}} \int\left|T_{f b, i a}^{(\mathrm{sw})}\right|^{2} d \hat{\mathbf{k}}_{f},
\end{aligned}
$$

where $\left[j_{a}\right]=\left(2 j_{a}+1\right)$ and

$$
\begin{aligned}
T_{f b, i a}^{(\mathrm{sw})} \equiv & \left\langle\psi_{\mathbf{k}_{f} m_{\mathrm{S} f}^{(-)}}^{(0)} \psi_{\epsilon_{b} \kappa_{b} m_{b}}(1)\left|\mathcal{H}_{\mathrm{int}}(0,1)\right|\right. \\
& \left.\times \psi_{\mathbf{k}_{i} m_{\mathrm{S} i}}^{(+)}(0) \psi_{n_{a} \kappa_{a} m_{a}}(1)\right\rangle
\end{aligned}
$$

is the "spherical" $T$-matrix element, which involves a central-field orbital $\psi_{\epsilon_{b} \kappa_{b} m_{b}}$ (1) of type (5) instead of a distorted wave for the ejected electron.
Using the well-known expansion of the Coulomb potential in terms of spherical Racah tensors [22],

$$
\frac{1}{\left|\mathbf{r}_{0}-\mathbf{r}_{1}\right|}=\sum_{L=0}^{\infty} \frac{r_{<}^{L}}{r_{>}^{L+1}} \mathbf{C}^{(L)}\left(\hat{\mathbf{r}}_{0}\right) \cdot \mathbf{C}^{(L)}\left(\hat{\mathbf{r}}_{1}\right)
$$

with $r_{<}=\min \left(r_{0}, r_{1}\right)$ and $r_{>}=\max \left(r_{0}, r_{1}\right)$, and elementary angular momentum algebra, it is possible to reduce the matrix elements to a relatively simple form. The final expression for the DCS is

$$
\begin{aligned}
\frac{d \sigma_{\mathrm{ion}}}{d W}= & \frac{2 Z_{0}^{2} e^{4}}{\hbar v_{i}} \frac{\left(\epsilon_{f}+2 m_{\mathrm{e}} c^{2}\right)\left(\epsilon_{b}+2 m_{\mathrm{e}} c^{2}\right)}{c^{4} \hbar^{4} k_{i}^{2} k_{f} k_{b}} \frac{\epsilon_{i}+2 m_{\mathrm{e}} c^{2}}{\epsilon_{i}+m_{\mathrm{e}} c^{2}} \\
& \times \sum_{\kappa_{b}} \sum_{\kappa_{i}} \sum_{\kappa_{f}} \sum_{L} \frac{1}{[L]}\left(X_{\epsilon_{f} \kappa_{f}, \epsilon_{b} \kappa_{b}, L}^{\epsilon_{i} \kappa_{i}, n_{a} \kappa_{a}}\right)^{2},
\end{aligned}
$$

where

$$
\begin{aligned}
X_{\epsilon_{f} \kappa_{f}, \epsilon_{b} \kappa_{b}, L}^{\epsilon_{i} \kappa_{i}, n_{a} \kappa_{a}}= & v\left(L, \ell_{f}, \ell_{i}\right) v\left(L, \ell_{b}, \ell_{a}\right) \sqrt{\left[j_{a}, j_{b}, j_{i}, j_{f}\right]} \\
& \times R_{\epsilon_{f} \kappa_{f}, \epsilon_{b} \kappa_{b}, L}^{\epsilon_{\epsilon_{i}}, n_{a} \kappa_{a}}\left(\begin{array}{rrr}
L & j_{i} & j_{f} \\
0 & \frac{1}{2} & -\frac{1}{2}
\end{array}\right) \\
& \times\left(\begin{array}{ccc}
L & j_{a} & j_{b} \\
0 & \frac{1}{2} & -\frac{1}{2}
\end{array}\right) .
\end{aligned}
$$

Here, the symbols $(:::)$ denote $3 j$ vector coupling coefficients; we have used the abbreviation $[x, y, \ldots]=(2 x+1)$ $\times(2 y+1) \ldots$ and $v\left(\ell_{1}, \ell_{2}, \ell_{3}\right)=1$ if $\ell_{1}+\ell_{2}+\ell_{3}$ is even and $=0$ otherwise. The quantities $R_{\epsilon_{f} \kappa_{f}, \epsilon_{b} \kappa_{b}, L}^{\epsilon_{i} \kappa_{i}, n_{a} \kappa_{a}}$ are Slater integrals,

$$
\begin{aligned}
R_{\epsilon_{f} \kappa_{f}, \epsilon_{b} \kappa_{b}, L}^{\epsilon_{i} \kappa_{i}, n_{a} \kappa_{a}}= & \iint d r_{0} d r_{1} \frac{r_{<}^{L}}{r_{>}^{L+1}}\left[P_{\epsilon_{i} \kappa_{i}}\left(r_{0}\right) P_{\epsilon_{f} \kappa_{f}}\left(r_{0}\right)\right. \\
& \left.+Q_{\epsilon_{i} \kappa_{i}}\left(r_{0}\right) Q_{\epsilon_{f} \kappa_{f}}\left(r_{0}\right)\right]\left[P_{n_{a} \kappa_{a}}\left(r_{1}\right) P_{\epsilon_{b} \kappa_{b}}\left(r_{1}\right)\right. \\
& \left.+Q_{n_{a} \kappa_{a}}\left(r_{1}\right) Q_{\epsilon_{b} \kappa_{b}}\left(r_{1}\right)\right] .
\end{aligned}
$$

The total ionization cross section is

$$
\sigma_{\text {ion }}=\int_{\left|\epsilon_{a}\right|}^{W_{\max }} \frac{d \sigma_{\text {ion }}}{d W} d W
$$

where the integral extends over the allowed energy-transfer interval, from the ionization threshold $\left|\epsilon_{a}\right|$ up to $W_{\max }=\epsilon_{i}$.

The theory presented up to this point is appropriate to describe positron ionizing collisions (and of any other spin $\frac{1}{2}$ projectiles that are distinguishable from the electron). Positrons differ from electrons in two important features. First, since the electric charges have opposite signs, the distorting field $V_{\mathrm{P}}$ is repulsive for positrons and attractive for electrons. Of course, this difference is not accounted for in the PWBA, which is equivalent to the present theory with $V_{\mathrm{P}}$ set equal to 
zero. Second, a projectile electron is indistinguishable from the active target electron and, therefore, it can undergo exchange scattering. The description of exchange scattering is simple when the initial and final orbitals of the target and the projectile are mutually orthogonal. The problem is much more difficult to handle when the orthogonality is not guaranteed (see, e.g., Ref. [23]), as happens in PWBA calculations.

\section{Electron collisions}

To account for the exchange effects in the simplest possible way, we assume that the projectile "sees" the same field as the active electron, i.e., $V_{\mathrm{P}} \equiv V_{\mathrm{T}}$. The projectile spherical waves are then orthogonal to the orbitals of the target active electron. The effect of exchange is described by antisymmetrizing the initial and final states in the transition matrix elements; that is, the transition matrix elements (4) are replaced by

$$
T_{f i}^{\mathrm{el}}=\left\langle\mathcal{A}\left[\psi_{f}(0) \psi_{b}(1)\right]\left|\mathcal{H}_{\mathrm{int}}(0,1)\right| \mathcal{A}\left[\psi_{i}(0) \psi_{a}(1)\right]\right\rangle,
$$

where the operator $\mathcal{A}$ is the two-particle antisymmetrizer. As the interaction is symmetrical, and the four orbitals are mutually orthogonal, the $T$-matrix element for electrons reduces to the following two terms:

$$
\begin{aligned}
T_{f i}^{(\mathrm{el})} \equiv & \left\langle\psi_{\mathbf{k}_{f} m_{\mathrm{S} f}^{(-)}}^{(0)} \psi_{\epsilon_{b} \kappa_{b} m_{b}}(1)\left|\mathcal{H}_{\mathrm{int}}(0,1)\right|\right. \\
& \left.\times \psi_{\mathbf{k}_{i} m_{\mathrm{S} i}}^{(+)}(0) \psi_{n_{a} \kappa_{a} m_{a}}(1)\right\rangle \\
& -\left\langle\psi_{\mathbf{k}_{f} m_{\mathrm{Sf}}^{(-)}}(1) \psi_{\epsilon_{b} \kappa_{b} m_{b}}(0)\left|\mathcal{H}_{\mathrm{int}}(0,1)\right|\right. \\
& \left.\times \psi_{\mathbf{k}_{i} m_{\mathrm{S} i}^{(+)}}(0) \psi_{n_{a} \kappa_{a} m_{a}}(1)\right\rangle,
\end{aligned}
$$

which describe direct and rearrangement transitions, respectively. The expression for the exchange term is derived, following the same scheme as for the direct term. The resulting DCS is

$$
\begin{aligned}
\frac{d \sigma_{\text {ion }}^{\mathrm{el}}}{d W}= & \frac{2 e^{4}}{\hbar v_{i}} \frac{\left(\epsilon_{f}+2 m_{\mathrm{e}} c^{2}\right)\left(\epsilon_{b}+2 m_{\mathrm{e}} c^{2}\right)}{c^{4} \hbar^{4} k_{i}^{2} k_{f} k_{b}} \frac{\epsilon_{i}+2 m_{\mathrm{e}} c^{2}}{\epsilon_{i}+m_{\mathrm{e}} c^{2}} \\
& \times \sum_{\kappa_{i}} \sum_{\kappa_{f}} \sum_{\kappa_{b}}\left[\sum_{L} \frac{1}{[L]}\left(X_{\epsilon_{f} \kappa_{f}, \epsilon_{b} \kappa_{b}, L}^{\epsilon_{i} \kappa_{i}, n_{a} \kappa_{a}}\right)^{2}\right. \\
& +\sum_{L^{\prime}} \frac{1}{\left[L^{\prime}\right]}\left(X_{\epsilon_{b} \kappa_{b}, \epsilon_{f} \kappa_{f}, L^{\prime}}^{\epsilon_{i} \kappa_{1}, n_{a} \kappa_{a}}\right)^{2} \\
& -2 \sum_{L} \sum_{L^{\prime}}(-1)^{L+L^{\prime}+1}\left\{\begin{array}{ccc}
j_{a} & j_{b} & L \\
j_{i} & j_{f} & L^{\prime}
\end{array}\right\} \\
& \left.\times X_{\epsilon_{f} \kappa_{f}, \epsilon_{b} \kappa_{b}, L}^{\epsilon_{i} \kappa_{i}, n_{a} \kappa_{a}} X_{\epsilon_{b} \kappa_{b}, \epsilon_{f} \kappa_{f}, L^{\prime}}^{\epsilon_{i} \kappa_{i}, n_{a} \kappa_{a}}\right],
\end{aligned}
$$

where $X_{\epsilon_{b} \kappa_{b}, \epsilon_{f} \kappa_{f}, L^{\prime}}^{\epsilon_{i} \kappa_{i}, n_{1} \kappa_{a}}$ is the $X$ coefficient, Eq. (16), corresponding to the exchange $T$-matrix element

$$
\begin{aligned}
X_{\epsilon_{b} \kappa_{b}, \epsilon_{f} \kappa_{f}, L^{\prime}}^{\epsilon_{i} \kappa_{i}, n_{k} \kappa_{a}}= & v\left(L^{\prime}, \ell_{b}, \ell_{i}\right) v\left(L^{\prime}, \ell_{f}, \ell_{a}\right) \sqrt{\left[j_{a}, j_{f}, j_{i}, j_{b}\right]} \\
& \times R_{\epsilon_{b} \kappa_{b}, \epsilon_{f} \kappa_{f}, L^{\prime}}^{\epsilon_{i} \kappa_{i}, n_{a} \kappa_{a}}\left(\begin{array}{rrr}
L^{\prime} & j_{i} & j_{b} \\
0 & \frac{1}{2} & -\frac{1}{2}
\end{array}\right) \\
& \times\left(\begin{array}{rrr}
L^{\prime} & j_{a} & j_{f} \\
0 & \frac{1}{2} & -\frac{1}{2}
\end{array}\right),
\end{aligned}
$$

which is obtained from Eq. (16) by means of the replacement $f \leftrightarrow b$. Note that, owing to the indistinguishability of the electrons in the final channel, the maximum allowed energy transfer is

$$
W_{\max }=\left(\epsilon_{i}+\left|\epsilon_{a}\right|\right) / 2
$$

\section{INTERACTION POTENTIALS}

Our formulation is based explicitly on the assumption that the active electron and the projectile move under the influence of local potentials $V_{\mathrm{T}}(r)$ and $V_{\mathrm{P}}(r)\left(=V_{\mathrm{T}}\right.$ if the projectile is an electron). To simplify the description of the present DWBA calculations, we shall set the target electron potential $V_{\mathrm{T}}$ equal to the self-consistent, spherically averaged, DiracFock-Slater (DFS) field, $V^{(\mathrm{DFS})}(r)$, which is completely determined by the density $\rho(r)$ of the atomic electrons. The DFS potential is given by

$$
V^{(\mathrm{DFS})}(r)=-e \varphi_{\mathrm{nuc}}(r)-e \varphi_{\mathrm{el}}(r)+V_{\mathrm{ex}}^{(\text {Slater })}(r),
$$

where $\varphi_{\text {nuc }}(r)=Z e / r$ is the electrostatic potential of the nucleus,

$$
\varphi_{\mathrm{el}}(r)=-\frac{e}{r} \int_{0}^{r} \rho\left(r^{\prime}\right) 4 \pi r^{\prime 2} d r^{\prime}-e \int_{r}^{\infty} \rho\left(r^{\prime}\right) 4 \pi r^{\prime} d r^{\prime}
$$

is the electrostatic potential of the atomic electron cloud, and

$$
V_{\mathrm{ex}}^{(\text {Slater })}(r)=-e^{2}(3 / \pi)^{1 / 3}[\rho(r)]^{1 / 3}
$$

is Slater's local approximation to the exchange interaction. To reproduce the correct large- $r$ behavior of the potential, $-(Z-N+1) e^{2} / r$, we adopt Latter's ad hoc prescription $[24,25]$ and define

$$
\begin{aligned}
& V^{(\mathrm{DFS})}(r) \\
& \equiv \begin{cases}-e \varphi_{\mathrm{nuc}}(r)-e \varphi_{\mathrm{el}}(r)+V_{\mathrm{ex}}^{(\text {Slater })}(r) & \text { if } r<r_{\text {Latter }}, \\
-(Z-N+1) e^{2} / r & \text { if } r>r_{\text {Latter }},\end{cases}
\end{aligned}
$$

where the cutoff radius $r_{\text {Latter }}$ is the outer root of the equation 


$$
-e \varphi_{\mathrm{nuc}}(r)-e \varphi_{\mathrm{el}}(r)+V_{\mathrm{ex}}^{(\text {Slater })}(r)=-(Z-N+1) e^{2} / r .
$$

The atomic electron orbitals $\psi_{n \kappa m}(\mathbf{r})$ satisfy the oneelectron Dirac equation (6) with the DFS potential. We assume that the one-electron eigenvalue $\epsilon_{n \kappa}$ is approximately equal to the ionization energy of the shell $(n, \kappa)$. In reality, experimental shell ionization energies [26] differ slightly from the corresponding one-electron DFS eigenvalues, and this introduces a certain error in the calculated cross sections. Fortunately, a large fraction of this error can be eliminated by simply rescaling the energy axis. Note, however, that such a correction has not been applied to the calculation results presented below.

As indicated above, for the electron scattering, we consider $V_{\mathrm{T}}(r)=V_{\mathrm{P}}(r)=V^{(\mathrm{DFS})}(r)$ so that the orbitals of the projectile and the active target electron are mutually orthogonal. This choice amounts to assuming that the projectile and target electrons interact with the inactive (spectator) atomic electrons in the same way, which is a plausible assumption. Note however, that this disregards the dependence of the exchange interaction on the electron velocity. For the positron scattering, it seems natural to take $V_{\mathrm{P}}(r)$ equal to the electrostatic interaction energy with the atomic charge distribution,

$$
V_{\mathrm{st}}(r)=e \varphi_{\mathrm{nuc}}(r)+e \varphi_{\mathrm{el}}(r) .
$$

Unfortunately, this potential reaches its asymptotic form $(=0)$ at distances that are much larger than $r_{\text {Latter }}$, and this makes the numerical computation of the ionization cross section much more difficult and lengthier than for electrons (see below). To circumvent this difficulty, we take $V_{\mathrm{P}}(r)=$ $-V^{(\mathrm{DFS})}(r)$, i.e., the distorting field is assumed to be the same as for an electron, but with the opposite sign. This field includes exchange contributions, which may seem inappropriate for a positron. Nonetheless, at large radial distances, the potential $-V^{(\mathrm{DFS})}(r)$ does represent the interaction of the positron with the nucleus and the spectator atomic electrons. Note that a part of the local exchange potential serves to eliminate the self-interaction of the atomic electrons (i.e., the interaction energy with their own charge distributions); a similar term must be subtracted from the electrostatic potential (28) to give the effective interaction of the positron with the inactive atomic charges.

The characteristics of the numerical algorithms employed in calculating inelastic cross sections are largely independent of the details of the adopted interaction potentials. In principle, we can expect to obtain more reliable results by using a more sophisticated atomic-structure model. In practice, however, the response of inner shells is determined by the innermost part of the atomic electron distribution, which is well described even by the simple DFS model.

\section{NUMERICAL ASPECTS}

The theory presented in Sec. II has been implemented in a FORTRAN 77 computer program named DWION, which calculates ionization cross sections of closed inner shells of atoms and positive ions by the impact of electrons and positrons. In this section, we comment on the essential features of the computer code; a detailed description of the numerical algorithm will be published elsewhere.

The radial wave functions of the bound and free orbitals involved are calculated using the subroutine package RADIAL developed by Salvat et al. [27], which implements a piecewise power-series solution method. The procedure consists of replacing the potential function $r V(r)$ by the interpolating natural cubic spline, and then evaluating the solution of the radial Dirac equations in each grid interval by summing its exact series expansion up to the required accuracy. The main advantage of the power-series method in front of more conventional solution methods (e.g., Runge-Kutta and predictorcorrector methods) is that truncation errors are effectively eliminated. The numerical solution is extended from the origin up to a certain radial distance $r_{\mathrm{m}}$, where the potential function $r V(r)$ has reached its asymptotic (constant) value. For $r>r_{\mathrm{m}}$, the radial functions can be expressed as a linear combination of the regular and irregular Dirac Coulomb functions, i.e., the exact solutions of the radial Dirac Equations (7) for a Coulomb field. RADIAL delivers nominally exact Dirac Coulomb functions, which are evaluated from their analytical expressions in terms of nonrelativistic Coulomb functions. Therefore, the numerical outward integration can be discontinued at a radius $r_{\mathrm{m}}$ (usually equal to $r_{\text {Latter }}$ ), where the value of the potential $V(r)$ is still appreciable. This not only saves computer time but also yields superior accuracy.

Vector coupling coefficients are evaluated directly from their analytical formulas, as given, e.g., by Rose [20]. The straight implementation of these formulas in a FORTRAN 77 code gives very inaccurate results for coefficients with moderately large quantum numbers. These coefficients are typically much less than unity, and are calculated as the sum of a finite series whose terms alternate in sign and, therefore, the result is directly affected by roundoff errors. These may mask the sought-for coefficient value completely, even when using double precision arithmetic. We have written a subroutine library that computes vector coupling coefficients (Clebsch-Gordan, $3 j$ and $6 j$ coefficients) using a radix-1000 representation of real numbers with 32 radix-1000 digits, which is equivalent to using a decimal representation with 96 digits. In the course of the calculation, roundoff errors are controlled very strictly to ensure that the final coefficient value, delivered as a double precision constant, is exact (within double precision accuracy, $\sim 15$ decimal digits). This has been verified by checking that the calculated coefficients satisfy various orthogonality relations.

Due to the large number of contributions from the four or fivefold summations, it is important to take advantage of the selection rules imposed by the angular factors, which considerably reduce the number of terms to be effectively calculated. The sums are performed in the order indicated in Eq. (15) or Eq. (21). For given values of $\kappa_{i}$ and $\kappa_{f}$, the allowed values for $\kappa_{b}, L$, and $L^{\prime}$ are determined by triangle inequalities and parity considerations, and the summations are carried out over the complete range of indices for which we have nonzero contributions. The summation over $\kappa_{f}$ is evaluated for increasing values of $\tau_{f}=\left|\kappa_{f}\right|$, until the combined 
contributions of the terms with $\kappa_{f}=\tau_{f}$ and $\kappa_{f}=-\tau_{f}$ amount to less than a small fraction of the accuracy required for the whole calculation (0.005 times the tolerance). Finally, the summation over $\kappa_{i}$ is calculated for increasing values of $\tau_{i}$ $=\left|\kappa_{i}\right|$ by adding the combined contributions of the terms with $\kappa_{i}=\tau_{i}$ and $\kappa_{i}=-\tau_{i}$. This summation is discontinued, and the calculation ended, when the relative contribution from the last added pair of terms is less than 0.05 times the tolerance. The maximum considered value of $\tau_{i}$ and $\tau_{f}$ is 200 , which has been proven to be large enough for calculating DCSs for projectiles with kinetic energies up to 9-10 times the ionization energy.

As partial-wave series are slowly convergent, the calculation of the DCSs involves the evaluation of a large number of Slater integrals of the form (17). Moreover, because of the highly oscillatory character of the integrand and the long range of the Coulomb interaction, the evaluation of these integrals is difficult and takes a very large fraction of the computation time. To optimize this evaluation, we use a method similar to the one described by Hartree [28] for bound states. We write the radial integral (17) as

$$
\begin{aligned}
I_{L} \equiv & R_{\epsilon_{f} \epsilon_{f}, \epsilon_{b} \kappa_{b}, L}^{\epsilon_{i} \kappa_{i}, n_{a} \kappa_{a}} \\
\underbrace{}_{0} & =\int_{0}^{\infty} d r g(r)\left\{\int_{0}^{r} \frac{s^{L}}{r^{L+1}} f(s) d s\right. \\
& \left.+\int_{r}^{\infty} \frac{r^{L}}{s^{L+1}} f(s) d s\right\},
\end{aligned}
$$

with

$$
f(s)=P_{n_{a} \kappa_{a}}(s) P_{\epsilon_{b} \kappa_{b}}(s)+Q_{n_{a} \kappa_{a}}(s) Q_{\epsilon_{b} \kappa_{b}}(s)
$$

and

$$
g(r)=P_{\epsilon_{i} \kappa_{i}}(r) P_{\epsilon_{f} \kappa_{f}}(r)+Q_{\epsilon_{i} \kappa_{i}}(r) Q_{\epsilon_{f} \kappa_{f}}(r) .
$$

Note that $f(s)$ involves the radial functions of the initial bound orbital. Therefore, $f(s)$ vanishes for $s$ greater than the "active shell radius" $r_{\mathrm{a}}$, defined as the distance at which these radial functions effectively vanish. Following Hartree [28], the function $Y(r)$, defined as

$$
Y(r)=\left\{\int_{0}^{r}\left(\frac{s}{r}\right)^{L} f(s) d s+\int_{r}^{\infty}\left(\frac{r}{s}\right)^{L+1} f(s) d s\right\},
$$

is determined by solving two differential equations, which is much faster than performing an integral for each $r$ in the grid.

The integral

$$
I_{L}=\int_{0}^{\infty} g(r) \frac{1}{r} Y(r) d r
$$

is evaluated in two steps. First, the integrand is tabulated at the points of a nonuniform grid that spans the interval $\left(0, r_{\mathrm{a}}\right)$ and that, by means of a simple change of variable, transforms into a uniform grid. The integral over the inner inter- val $\left(0, r_{\mathrm{a}}\right)$ is then calculated by using the six-point Lagrange quadrature rule. For large enough radii, the radial functions are expressed as linear combinations of regular and irregular Dirac Coulomb functions. Using the known asymptotic expansions of the latter [27], we can write

$$
\begin{aligned}
& P_{\epsilon \kappa}(r)=\mathcal{A}_{1}(r) \cos \phi_{\epsilon \kappa}+\mathcal{A}_{2}(r) \sin \phi_{\epsilon \kappa}, \\
& Q_{\epsilon \kappa}(r)=\mathcal{A}_{3}(r) \cos \phi_{\epsilon \kappa}+\mathcal{A}_{4}(r) \sin \phi_{\epsilon \kappa},
\end{aligned}
$$

with

$$
\begin{aligned}
\phi_{\epsilon \kappa}= & k r-\eta \ln r+\left[\delta_{\epsilon \kappa}+\arg \Gamma\left(\lambda_{i}+\mathrm{i} \eta\right)\right. \\
& \left.-\eta \ln (2 k)-(\lambda-1) \frac{\pi}{2}\right]
\end{aligned}
$$

where $\eta$ is the Sommerfeld parameter and the functions $\mathcal{A}_{j}(r)$ are defined as power series of $r^{-1}$, with coefficients that are obtained analytically. It is assumed that these asymptotic expansions converge for $r>r_{\mathrm{a}}$; otherwise, we simply increase the value of $r_{\mathrm{a}}$ to fulfill this condition. Thus, the integral over the outer interval $\left(r_{\mathrm{a}}, \infty\right)$ is reduced to the form

$$
I_{L}^{\text {out }} \equiv \int_{r_{\mathrm{a}}}^{\infty} g(r) \frac{1}{r} Y(r) d r=\sum_{n=0}^{\infty} \int_{r_{\mathrm{a}}}^{\infty}\left\{\mathcal { A } _ { \mathrm { s } , n } \operatorname { s i n } \left(b_{\mathrm{s}, n} r-c_{\mathrm{s}, n} \ln r\right.\right.
$$

$$
\left.\left.+d_{\mathrm{s}, n}\right)+\mathcal{A}_{\mathrm{c}, n} \cos \left(b_{\mathrm{c}, n} r-c_{\mathrm{c}, n} \ln r+d_{\mathrm{c}, n}\right)\right\} \frac{1}{r^{n+L+1}} d r,
$$

where $A_{\mathrm{s}, n}, b_{\mathrm{s}, n}, \ldots$ are constants defined by analytical expressions and recurrence relations. The integrals in this expression are the real and imaginary parts of the following:

$$
\begin{aligned}
& \int_{r_{\mathrm{a}}}^{\infty} \exp [\mathrm{i}(b r-c \ln r+d)] \frac{1}{r^{n+L+1}} d r \\
& \quad=\mathrm{e}^{\mathrm{i} d}(-\mathrm{i} b)^{n+L+\mathrm{i} c} \Gamma\left(-n-L-\mathrm{i} c,-\mathrm{i} b r_{0}\right),
\end{aligned}
$$

where $\Gamma(\alpha, z)$ is the incomplete gamma function with complex arguments [29],

$$
\Gamma(\alpha, z) \equiv z^{\alpha} \int_{1}^{\infty} \mathrm{e}^{-z u} u^{\alpha-1} d u .
$$

In our computer code, $\Gamma(\alpha, z)$ is evaluated from its continued fraction representation [29],

$$
\Gamma(\alpha, z)=\mathrm{e}^{-z} z^{\alpha}\left(\frac{1}{z+} \frac{1-a}{1+} \frac{1}{z+} \frac{2-a}{1+} \frac{2}{z+} \frac{3-a}{1+} \cdots\right),
$$

which yields nominally exact (FORTRAN double precision) values. With all this, the outer integral $I_{L}^{\text {out }}$ can be evaluated 
as a sum of integrals given by closed analytical expressions. In practice, a convergence is usually attained after adding about $n=50$ terms.

Our computer code automatically determines the minimum value of the cutoff radius $r_{\mathrm{a}}$ for which the asymptotic expansions of free Coulomb wave functions converge. We have checked the consistency of the whole calculation by just comparing the results obtained with different values of the cutoff radius $r_{\mathrm{a}}$. The stability of the algorithm is such that, in typical cases, a variation of $r_{\mathrm{a}}$ by a factor of the order of 2-5 induces changes in the sixth decimal digit of the calculated DCSs. Therefore, the numerical relative error of the DCSs is expected to be of the order of $0.01 \%$ or less. The calculation method works provided only that enough computer time and memory storage are available.

It is worth mentioning that the numerical algorithm used to calculate the radial wave functions of free states is not applicable when the kinetic energy of the particle is too small. The reason is that, to normalize the state, numerical radial functions have to be matched at $r_{\mathrm{m}}$ to a linear combination of regular and irregular Dirac Coulomb functions. Irregular Coulomb functions increase without limit when the radius $r$ decreases below the classical turning point. When the kinetic energy is small and the orbital angular momentum $\ell$ increases, the turning point moves away from the origin and the numerical integration procedure must be applied for wider and wider $r$ intervals. Eventually, it becomes too inefficient and we need to avoid calculations involving low kinetic energies. This occurs for energy losses $W$ near the ionization energy (the ejected electron is slow) and, in the case of positrons, for $W \sim \epsilon_{i}$ (the positron slows down to rest). To avoid computing these extreme cases, our code uses an adaptive algorithm to determine the $W$ values for which the DCS needs to be calculated. In the initial stage, the DCS is calculated for a coarse uniform grid with approximately ten points that span the interval from $W=\left|\epsilon_{a}\right|$ to $W_{\max }$ excluding the end points. This grid is used to set a natural cubic spline that interpolates $\ln \left(d \sigma_{\text {ion }} / d W\right)$ as a function of $W$. The DCS is then evaluated at the midpoint of the subinterval where the spline has its largest curvature (including the first and last subintervals, where the spline is used to extrapolate the DCS) and a new interpolating spline is determined. The process continues until the integral of the DCS (i.e., of the interpolating spline) reaches its saturation value (to within the required tolerance). With this adaptive method, we need only to calculate the DCSs for $W$ values that are sufficiently far from the end points of the allowed interval to avoid the aforesaid numerical difficulty.

\section{Accuracy and stability of the calculations}

It is clear that the numerical calculation of the DWBA ionization DCS, as given by Eqs. (15) and (21), is not only very time consuming but also prone to be affected by accumulated numerical errors. To give an idea of the difficulty of these calculations, we would like to mention that to get a convergence of the series (15), we need to sum up as many as 50000 terms (or even more). Hence, it is essential to devise a rigorous method to test the absolute accuracy of the whole calculation performed by the program DWION. Unfortunately, there is no simple way to test DWBA calculations in which the projectile sees a nonvanishing field $V_{\mathrm{P}}(r)$. The only possible alternative is to run the code with $V_{\mathrm{P}}(r) \equiv 0$ and disregarding the exchange $T$ matrix in the case of electrons. In this case, the code should give results identical to those obtained from the PWBA, although it performs strictly the same operations as for a DWBA calculation.

The point is that PWBA calculations are much easier and robust because the integrals involving the projectile plane waves are calculated analytically. We have written a second computer program named PWION that calculates ionization DCSs using the PWBA, with exactly the same physical model as in the present DWBA calculations. To validate this code, calculations were performed for hydrogenic ions, with the speed of light $c$ multiplied by $10^{6}$. The results were found to agree to within six significant digits with the nonrelativistic PWBA DCS (which was calculated from the analytical generalized oscillator strength [30] by a single quadrature). The comparison of DCSs calculated with PWION and DWION with $V_{\mathrm{P}}=0$ and no exchange corrections for electrons then provides a stringent and unambiguous test of the numerical accuracy and stability of the DWION code.

Figure 2 displays DCSs for ionization of the $\operatorname{argon} K$ shell by the impact of electrons with various kinetic energies calculated according to the PWBA by the codes PWION and DWION, the latter with $V_{\mathrm{P}}=0$ and considering that the projectile is distinguishable from the target electron (i.e., with exchange corrections switched off). The DWION data (crosses) were automatically generated by the code using the adaptive procedure described above; these are unevenly spaced in $W$. The corresponding continuous DCS is obtained from this discrete set of values by natural cubic spline interpolation (extrapolation) of $\ln \left(d \sigma_{\text {ion }} / d W\right)$ as a function of $W$. The differences between DCSs and total cross sections resulting from the two calculations are less than the tolerance adopted in the DWION calculation $\left(10^{-3}\right)$. The insets in Fig. 2 show the relative difference between the DCSs calculated by the two codes, which is, in general, less than $0.1 \%$. Slightly larger differences are seen in the low-energy-loss region, where the spline is used to extrapolate to the ionization threshold. Similar comparisons of PWBA results from the DWION and PWION codes have been made for multiple cases with different atomic numbers, active electron shells, and projectile kinetic energies. The calculated DCSs and total cross sections always differed by less than the tolerance adopted in DWION.

We have also compared PWBA total cross sections calculated with both codes as functions of the kinetic energy of the projectile; the results are illustrated in Fig. 3 for the case of the $K$ shell of argon. Differences in total cross sections are, in general, below $0.1 \%$, except in the near-threshold range where the difference rises to $\sim 0.3 \%$. As mentioned above, DWION (and, to a lesser extent, also PWION) has difficulties to calculate cross sections for projectiles with kinetic energies near the ionization threshold. The crosses in Fig. 3 are data calculated by the DWION code. These extend down to energies very close to the threshold and, therefore, this limitation is not critical for practical purposes. The excellent 

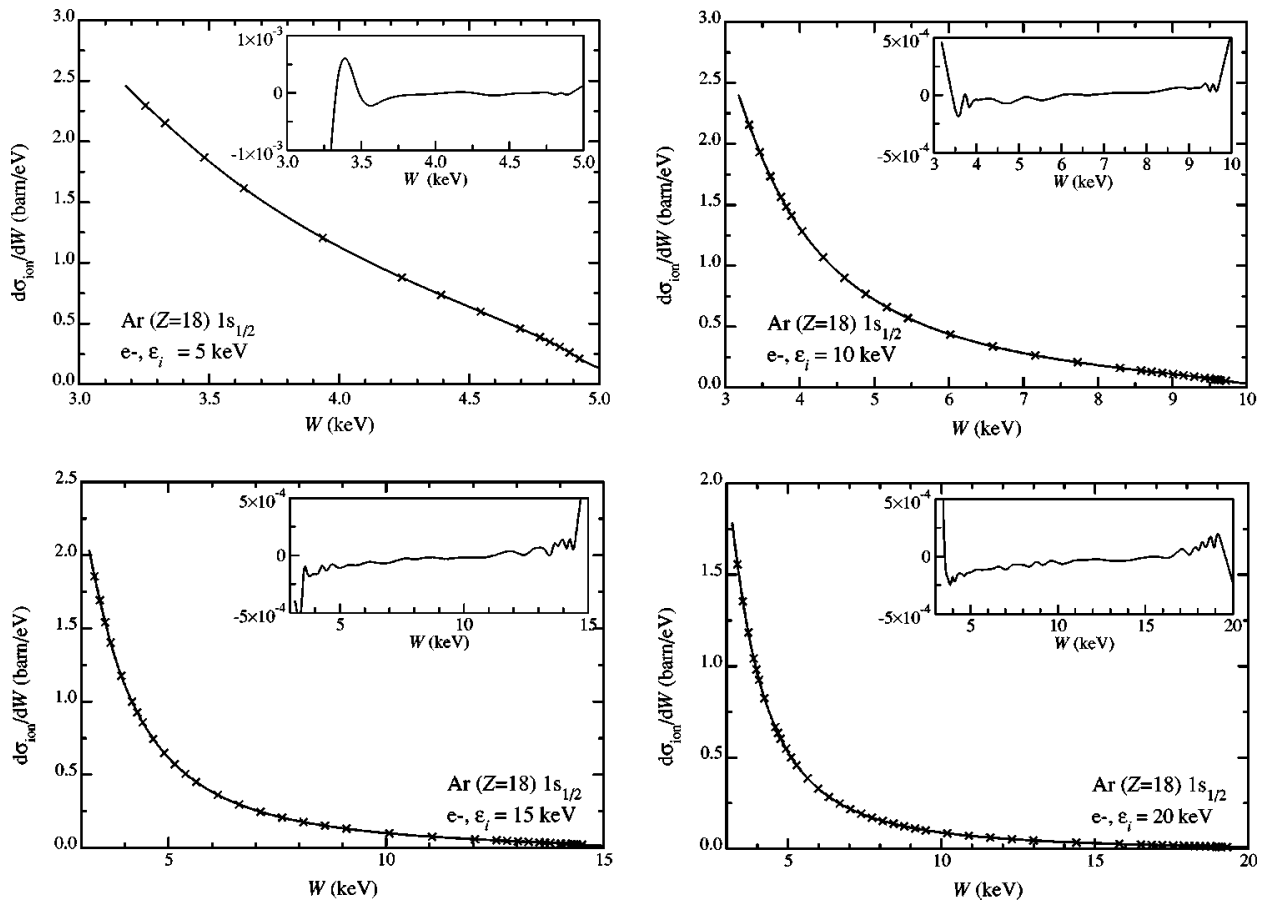

FIG. 2. The PWBA DCSs for ionization of the $K$ shell of argon, $\left|\epsilon_{a}\right|=3177.6 \mathrm{eV}$, by electrons with kinetic energy $\epsilon_{i}=5,10,15$, and $20 \mathrm{keV}$. The solid curves represent results from the conventional PWBA calculated by our program PWION. Crosses are DCS values generated by the distortedwave program DWION with the option $V_{\mathrm{P}}=0$ and no exchange. agreement between PWBA results from the two codes demonstrates the accuracy of the numerical algorithm implemented in DWION and, at the same time, confirms that our estimation and control of numerical errors are appropriate.

\section{RESULTS AND DISCUSSION}

The program DWION has been used to perform calculations of total cross sections for ionization of the $K$ and $L$ shells of neutral atoms by electron and positron impact. To assess the reliability of these calculations, we compare here our numerical results with experimental ionization cross sections. As mentioned in the introduction, experimental data are usually affected by considerable uncertainties, which are evident from the large differences between data from differ-

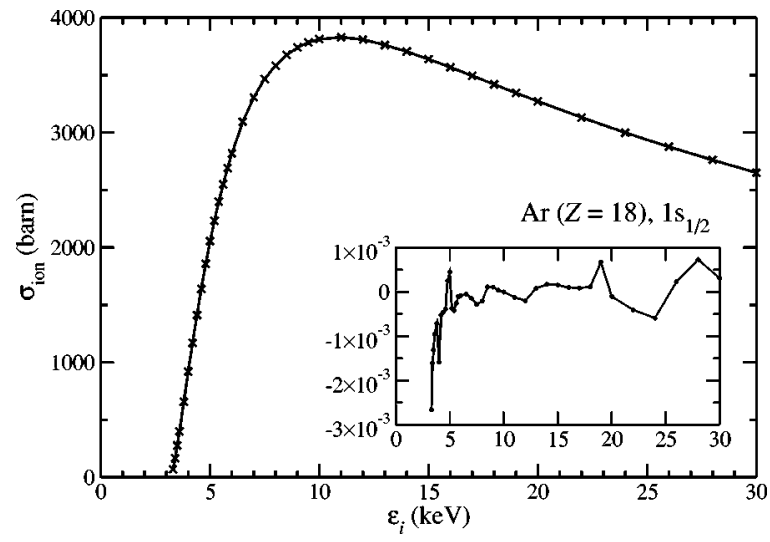

FIG. 3. PWBA total cross section for $K$-shell ionization of argon by the impact of electrons as a function of the kinetic energy $\epsilon_{i}$ of the projectile. Crosses are results from the DWION code with $V_{\mathrm{P}}$ $=0$ and no exchange correction; the solid curve represents results from the conventional PWBA calculated with our PWION code. The relative difference is shown in the inset. ent laboratories. Therefore, it is convenient to limit the comparison of calculation with experiment to those cases for which at least two independent measurements are available.

Figure 4 displays cross sections for ionization of the $K$ shell of $\operatorname{argon}(Z=18)$ by the electron impact. The calculated DWBA cross sections for electrons are seen to agree well with results from measurements reported in Refs. [31$34]$, the relative differences are of the order of $10 \%$.

In Figs. 5-9, we compare DWBA calculated cross sections for ionization of the $K$ shell of the elements chromium $(Z=24)$, manganese $(Z=25)$, iron $(Z=26)$, nickel $(Z$ $=28)$, and copper $(Z=29)$ with experimental data. For these transition metals, we rely mostly on ionization cross sections measured recently by our group $[15,16,35]$. These data were obtained by measuring $\mathrm{x}$ rays emitted from very thin targets in an electron microprobe. The x-ray intensities (i.e., relative cross sections) were affected by uncertainties of about $2 \%$.

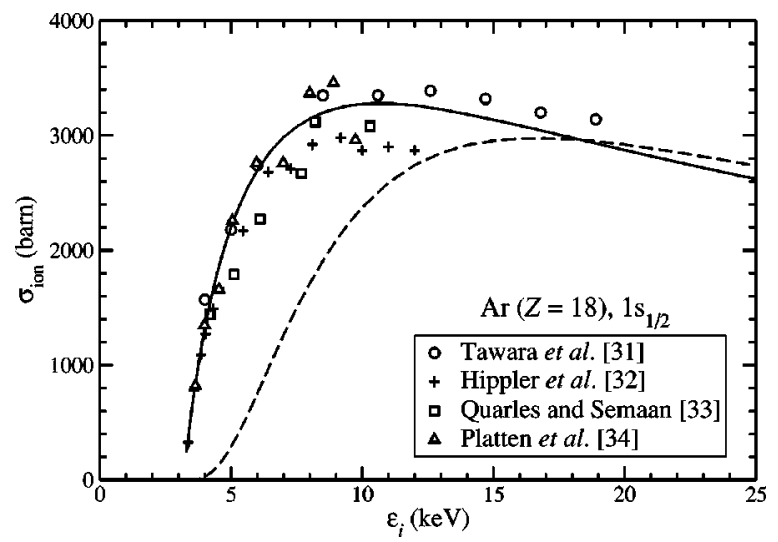

FIG. 4. Total cross sections for ionization of the $K$ shell of argon, $\left|\epsilon_{a}\right|=3177.64 \mathrm{eV}$, by electrons (solid curve) and positrons (dashed curve). Symbols represent experimental data [31-34] for the electron impact. 


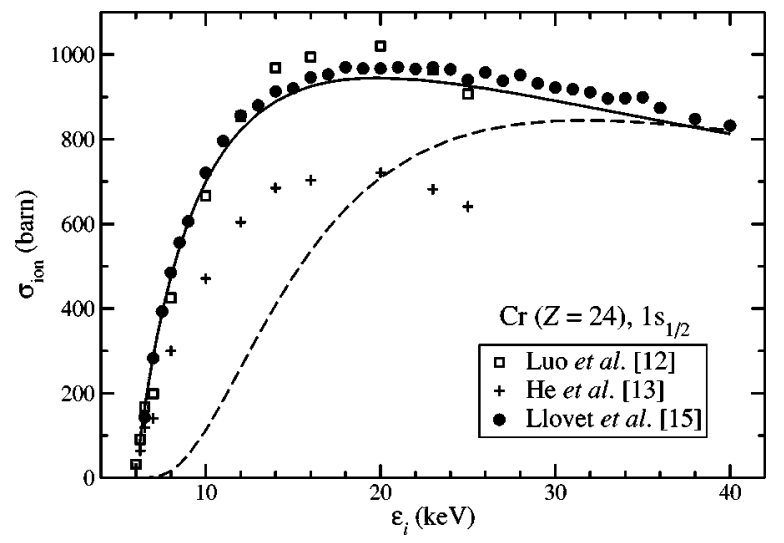

FIG. 5. Total cross sections for ionization of the $K$ shell of chromium, $\left|\epsilon_{a}\right|=5963.05 \mathrm{eV}$, by electrons (solid curve) and positrons (dashed curve). Symbols represent experimental data $[12,13,15]$ for the electron impact.

The conversion into absolute ionization cross-section values introduced additional systematic uncertainties. The estimated error reported for the experimental cross sections was $\sim 10-11 \%$. The differences between our calculations and the experimental data of Llovet et al. $[15,35]$ are, in general, less than this error estimate, except for copper where the average difference was $\sim 15 \%$. In all cases, however, the shape of the calculated cross section versus energy curve is consistent with experiment. To illustrate this fact, in Fig. 9, we have included the result of multiplying the calculated $\sigma_{\text {ion }}(E)$ curve by a constant factor of 1.15 (dot-dashed curve). The agreement with the measured data is seen to be excellent. Therefore, the observed differences between experimental absolute values and DWBA calculations are very likely attributable to the systematic uncertainties introduced in the conversion of measured $\mathrm{x}$-ray intensities into absolute cross-section values. This seems also to be true for experimental data from other authors, which appear to differ from the DWBA results by a roughly constant factor.

Calculated ionization cross sections for the $K$ shell of silver by electron impact are shown in Fig. 10, together with the experimental data from three different publications. Note

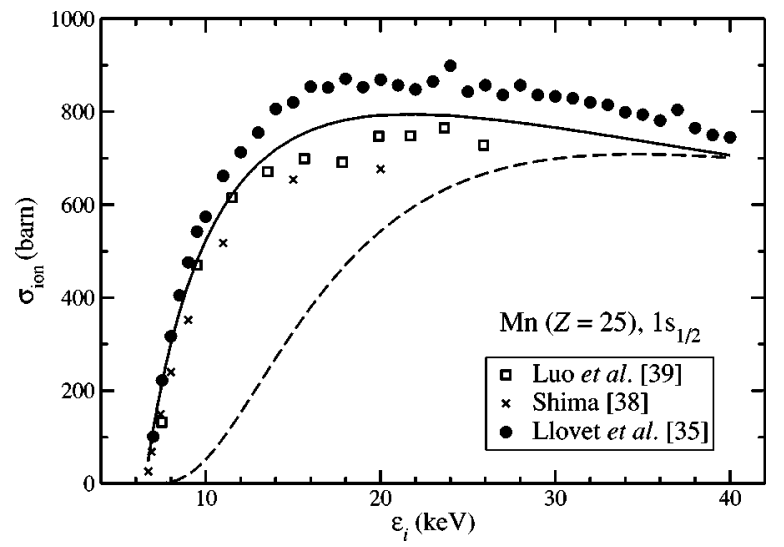

FIG. 6. Total cross sections for ionization of the $K$ shell of manganese, $\left|\epsilon_{a}\right|=6510.94 \mathrm{eV}$, by electrons (solid curve) and positrons (dashed curve). Symbols represent experimental data $[35,38,39]$ for the electron impact.

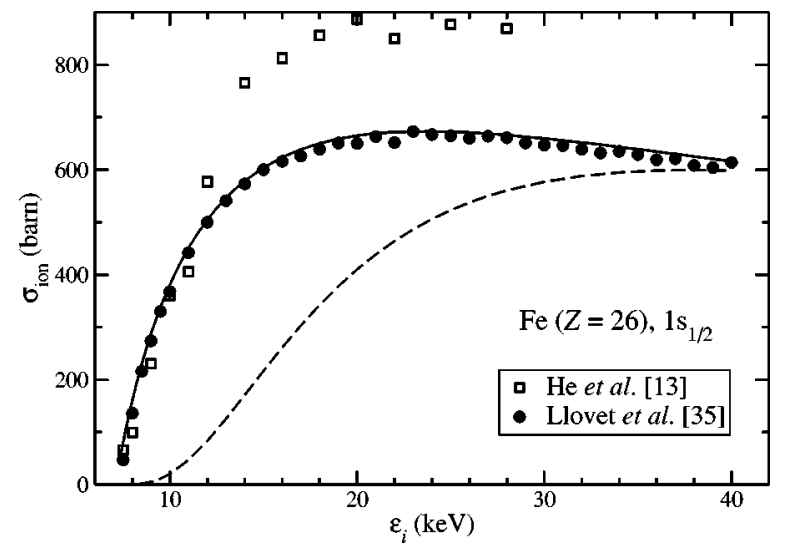

FIG. 7. Total cross sections for ionization of the $K$ shell of iron, $\left|\epsilon_{a}\right|=7083.48 \mathrm{eV}$, by electrons (solid curve) and positrons (dashed curve). Symbols represent experimental data $[13,35]$ for the electron impact.

that the shape of the theoretical curve is consistent with the data of Davis et al. [36], although the calculation is $\sim 25 \%$ lower than the experiment. The data of Schneider et al. [11] practically coincide with the theoretical curve, while the data from Hansen and Flammersfeld [37] do not follow the expected trend.

Cross sections for ionization of the $L_{3}$ shell of gold are shown in Fig. 11. Our calculation is seen to agree closely with data of Schneider et al. [11] in the near-threshold region. For higher incident energies the theory follows the experimental data of Davis et al. [36], while data of Schneider et al. decrease faster with increasing kinetic energy.

We would also like to mention that the DWION code was recently used by Campos et al. [16] to calculate $L_{\alpha}$ X-ray production cross sections $\sigma_{L \alpha}$ by electron impact on tungsten $(Z=74)$, platinum $(Z=78)$, and gold $(Z=79)$. The theoretical evaluation of $\sigma_{L \alpha}$ involves the ionization cross sections of the three $L$ subshells, which were calculated by the DWION code, and Koster-Kronig transition probabilities and x-ray emission rates [see Eq. (2) in Ref. [16]]. These authors also performed experimental measurements of $\sigma_{L \alpha}$ on an electron microprobe. Their data were found to be in excellent

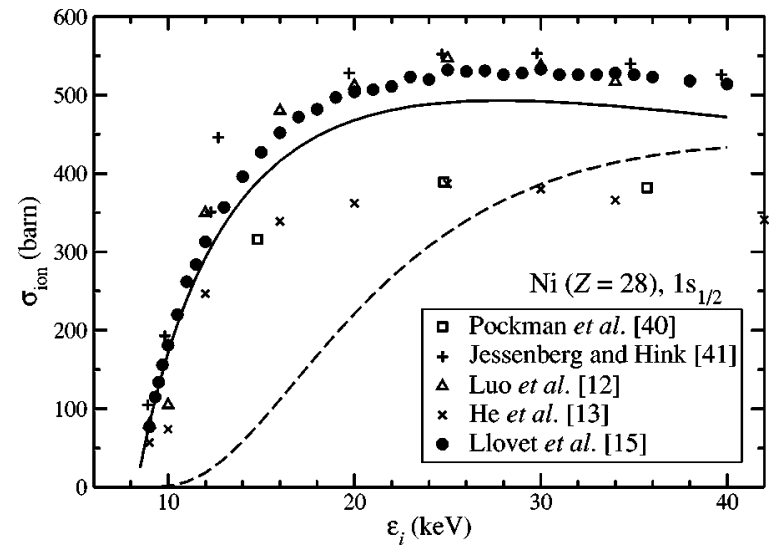

FIG. 8. Total cross sections for ionization of the $K$ shell of nickel, $\left|\epsilon_{a}\right|=8303.01 \mathrm{eV}$, by electrons (solid curve) and positrons (dashed curve). Symbols represent experimental data $[12,13,15,40,41]$ for the electron impact. 


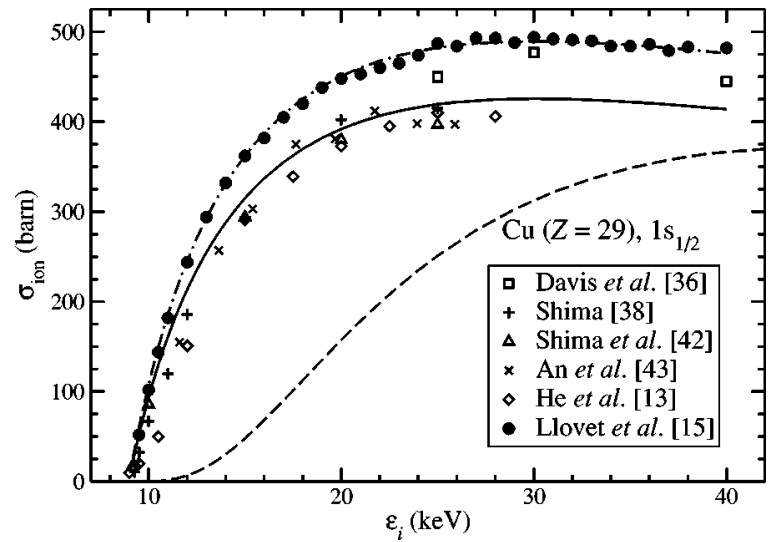

FIG. 9. Total cross sections for ionization of the $K$ shell of copper, $\left|\epsilon_{a}\right|=8950.26 \mathrm{eV}$, by electrons (solid curve) and positrons (dashed curve). Symbols represent experimental data $[13,15,36,38,42,43]$ for the electron impact. The dot-dashed curve is the result of multipliyng the DWBA cross section by a constant factor.

agreement with the theoretical DWBA calculations; the differences were always less than the estimated uncertainty of the measured data, which was of the order of $11 \%$.

As indicated above, the present DWBA calculations provide a consistent description of the differences between electron and positron collisions. These differences originate from the opposite charges of the projectiles (electrons are accelerated and positrons are slowed down by the atomic field) and also from exchange interactions (which occur only for electrons). These two characteristics are not accounted for by the PWBA, which predicts the same ionization cross sections for electrons and positrons.

In Figs. 4-11, we have also included calculated ionization cross sections for positron impact (dashed curves). Experimental absolute cross sections for positrons are very scarce. Schneider et al. [11] have reported absolute measurements for the $K$ shell of silver and the $L_{3}$ subshell of gold. Hansen

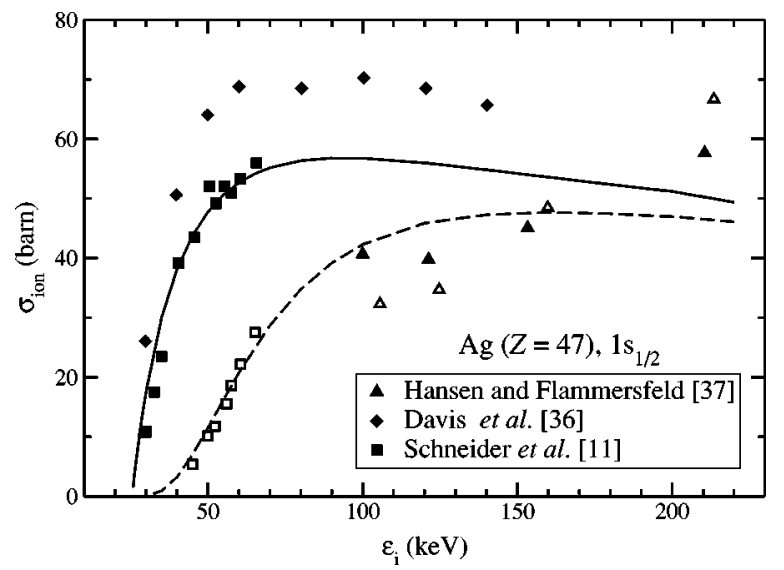

FIG. 10. Total cross sections for ionization of the $K$ shell of silver, $\left|\epsilon_{a}\right|=25489.79 \mathrm{eV}$, by electrons (solid curve) and positrons (dashed curve). Solid and open symbols represent experimental data for electrons and positrons, respectively; squares, Schneider et al. [11]; triangles, Hansen and Flammersfeld [37]; diamonds, Davis et al. [36].

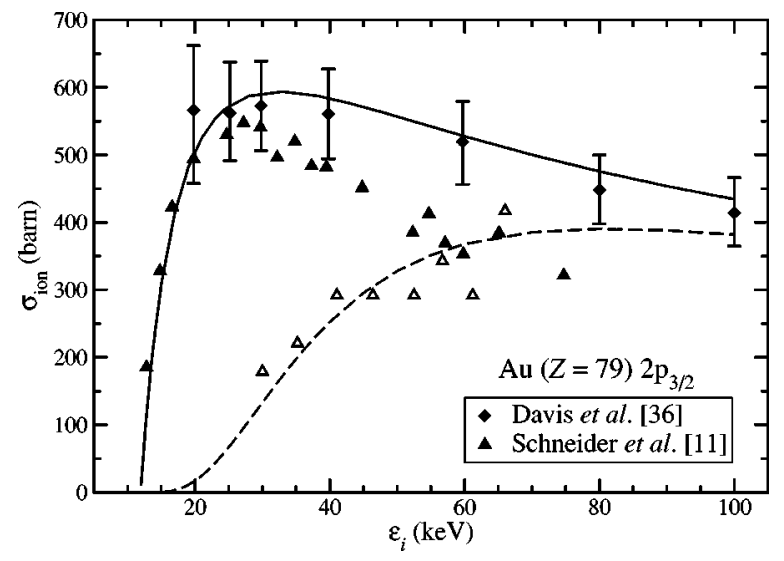

FIG. 11. Total cross sections for ionization of the $L_{3}$ shell of gold, $\left|\boldsymbol{\epsilon}_{a}\right|=11921.89 \mathrm{eV}$, by electrons (solid curve) and positrons (dashed curve). Solid and open symbols represent experimental data for electrons and positrons, respectively: triangles, Schneider et al. [11]; diamonds, Davis et al. [36].

and Flammersfeld [37] also published positron ionization cross sections for the $K$ shell of silver, in a higher-energy range (100-400 keV). These experimental data are displayed in Figs. 10 and 11. We see that the theoretical cross section for ionization of the silver $K$ shell by positron impact is in excellent agreement with the experimental data of Schneider et al.

Ito et al. [44] have reported the ratio $\sigma^{-} / \sigma^{+}$, i.e., the electron cross section relative to positron cross section, as a function of the overvoltage $U=\epsilon_{i} /\left|\epsilon_{a}\right|$. This ratio is relatively independent of the atomic number, it approximates unity for large overvoltages, at which the PWBA should be nearly correct. For projectiles with kinetic energy $\epsilon_{i}$ near the threshold, the ratio increases with decreasing $\epsilon_{i}$. It is interesting to note the marked differences between the crosssection curves for electrons and positrons at small overvoltages, which arise from the different distortions caused by the atomic field on projectiles with opposite charges. For large overvoltages, the effect of exchange dominates and the positron cross section becomes larger than the electron cross section (see Fig. 4).

In our calculations, the contribution of the transverse interaction to the ionization cross sections (see, e.g., Ref, [17]) has been disregarded. The effect of this interaction increases with the kinetic energy of the projectile and with the atomic number of the target atom. Calculations for ionization of the $K$ shell of gold (a quite unfavorable case) by electrons using the PWBA [9] with the complete interaction (but disregarding exchange effects) indicate that inclusion of the transverse interaction increases the total ionization cross section by about 3\% near the ionization threshold, which is at $80.7 \mathrm{keV}$. For projectiles with kinetic energy $\epsilon_{i}$ equal to $100 \mathrm{keV}$, the transverse contribution is $\sim 4 \%$ and increases to $\sim 9 \%$ at $\epsilon_{i}=200 \mathrm{keV}$. Therefore, when the energy of the projectile is smaller than $\sim 100 \mathrm{keV}$, inclusion of the transverse interaction in DWBA calculations would be needed only for the $K$ shell of heavy elements. In conclusion, the present formulation and calculation scheme provides a consistent description of the ionization of inner shells of neutral atoms by impact of 
electrons and positrons with kinetic energies up to about 100 $\mathrm{keV}$.

\section{ACKNOWLEDGMENTS}

We would like to thank J. M. Fernández-Varea for helpful comments and discussions including valuable ideas and as- sistance with the PWBA calculations and C. S. Campos for testing the DWION program. This work has been partially supported by the Spanish Fondo de Investigación Sanitaria (Project No. FIS 01/0093). S.S. thanks the CONICET (Argentina) for financial support and M.D. expresses his gratitude to the European Union, the Marie Curie Program (Grant No. FIGD-CT-1999-50002).
[1] R. Mayol and F. Salvat, J. Phys. B 23, 2117 (1990).

[2] R. Hippler, Phys. Lett. A 144, 81 (1990).

[3] C.J. Powell, in Electron Impact Ionization, edited by T.D. Märk and G.H. Dunn (Springer Verlag, New York, 1985), p. 198.

[4] E. Casnati, A. Tartari, and C. Baraldi, J. Phys. B 15, 155 (1982).

[5] C. Hombourger, J. Phys. B 31, 3693 (1998).

[6] S. Keller, C.T. Whelan, H. Ast, H.R.J. Walters, and R.M. Dreizler, Phys. Rev. A 50, 3865 (1994).

[7] D.H. Sampson, Phys. Rev. A 34, 986 (1986).

[8] C.J. Fontes, D.H. Sampson, and H.L. Zhang, Phys. Rev. A 47, 1009 (1993).

[9] J. M. Fernández-Varea, S. Segui, M. Dingfelder, and F. Salvat (unpublished).

[10] X. Long, M. Lu, F. Ho, and X. Peng, At. Data Nucl. Data Tables 45, 353 (1990).

[11] H. Schneider, I. Tobehn, F. Ebel, and R. Hippler, Phys. Rev. Lett. 71, 2707 (1993).

[12] Z.M. Luo, Z. An, F. He, T. Li, X. Long, and X. Peng, J. Phys. B 29, 4001 (1996).

[13] F.Q. He, X.F. Peng, X.G. Long, Z.M. Luo, and Z. An, Nucl. Instrum. Methods Phys. Res. B 129, 445 (1997).

[14] X. Peng, F. He, X. Long, Z.M. Luo, and Z. An, Phys. Rev. A 58, 2034 (1998).

[15] X. Llovet, C. Merlet, and F. Salvat, J. Phys. B 33, 3761 (2000).

[16] C.S. Campos, M.A.Z. Vasconcellos, X. Llovet, and F. Salvat, Phys. Rev. A 66, 012719 (2002).

[17] U. Fano, Annu. Rev. Nucl. Sci. 13, 1 (1963).

[18] Y. Itikawa, Phys. Rep. 148, 69 (1986).

[19] I.P. Grant, Adv. Phys. 19, 747 (1970).

[20] M.E. Rose, Relativistic Electron Theory (Wiley, New York, 1961).

[21] C.J. Joachain, Quantum Collision Theory (North-Holland, Amsterdam, 1983).

[22] A.R. Edmonds, Angular Momentum in Quantum Mechanics (Princeton University Press, Princeton, NJ, 1957).
[23] D.H. Madison and E. Merzbacher, in Atomic Inner-Shell Processes, edited by B. Crasemann (Academic Press, New York, 1975), pp. 1-72.

[24] R. Latter, Phys. Rev. 99, 510 (1955).

[25] D. Liberman, D.T. Cromer, and J.T. Waber, Comput. Phys. Commun. 2, 107 (1971).

[26] J.A. Bearden and A. Burr, Rev. Mod. Phys. 39, 125 (1967).

[27] F. Salvat, J.M. Fernández-Varea, and W. Williamson, Jr., Comput. Phys. Commun. 90, 151 (1995).

[28] D.R. Hartree, The Calculation of Atomic Structure (Wiley, New York, 1957).

[29] M. Abramowitz and I.A. Stegun, Handbook of Mathematical Functions (Dover, New York, 1964).

[30] M. Inokuti, Rev. Mod. Phys. 43, 297 (1971).

[31] H. Tawara, K.G. Harrison, and F.J. de Heer, Physica (Amsterdam) 63, 351 (1973).

[32] R. Hippler, H. Klar, K. Saeed, I. McGregor, H. Kleinpoppen, and A.J. Duncan, J. Phys. B 16, L617 (1983).

[33] C. Quarles and M. Semaan, Phys. Rev. A 26, 3147 (1982).

[34] H. Platten, G. Schiwietz, and G. Nolte, Phys. Lett. 107A, 83 (1985).

[35] X. Llovet, C. Merlet, and F. Salvat, J. Phys. B 35, 973 (2002).

[36] D.V. Davis, V.D. Mistry, and C.A. Quarles, Phys. Lett. 38A, 169 (1972).

[37] H. Hansen and A. Flammersfeld, Nucl. Phys. 79, 135 (1966).

[38] K. Shima, Phys. Lett. 77A, 237 (1980).

[39] Z.M. Luo, Z. An, T. Li, L. Wang, Q. Zhu, and X. Xia, J. Phys. B 30, 2681 (1997).

[40] L.T. Pockman, D.L. Webster, P. Kirkpatrick, and K. Harworth, Phys. Rev. 71, 330 (1947).

[41] J. Jessenberg and W. Hink, Z. Phys. A 275, 331 (1975).

[42] K. Shima, T. Nakagawa, K. Umetani, and T. Fikumo, Phys. Rev. A 24, 72 (1981).

[43] Z. An, T.H. Li, L.M. Wang, X.Y. Xia, and Z.M. Luo, Phys. Rev. A 54, 3067 (1996).

[44] S. Ito, S. Shimizu, T. Kawaratani, and K. Kubota, Phys. Rev. A 22, 407 (1980). 